\title{
Trophoblast Glycoprotein: Possible Candidate Mediating Podocyte Injuries in Glomerulonephritis
}

\author{
Taichi Murakami $^{a} \quad$ Hideharu Abe $^{a}$ Kojiro Nagai ${ }^{a}$ Tatsuya Tominaga ${ }^{a}$ \\ Norimichi Takamatsu ${ }^{b}$ Toshikazu Araoka ${ }^{a}$ Seiji Kishi ${ }^{a}$ Toshikazu Takahashia \\ Akira Mima $^{a}$ Yoshimi Takaic Jeffrey B. Kopp ${ }^{d}$ Toshio Doi ${ }^{\mathrm{a}}$ \\ aDepartment of Nephrology, Graduate School of Medicine, Institute of Health-Bio-Science, University of

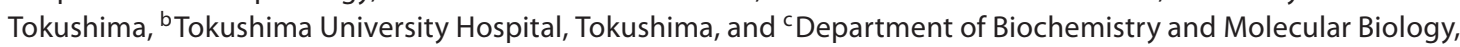 \\ Division of Molecular and Cellular Biology, Kobe University Graduate School of Medicine, Kobe, Japan; \\ ${ }^{\mathrm{d}}$ Kidney Disease Section, National Institute of Diabetes and Digestive and Kidney Diseases, National Institutes \\ of Health, Bethesda, Md., USA
}

\section{Key Words}

Trophoblast glycoprotein • Podocyte - Thy 1 glomerulonephritis - Transforming growth factor- $\beta$ • Cell motility · Stress fiber

\begin{abstract}
Background: Trophoblast glycoprotein (Tpbg), a 72-kDa transmembrane glycoprotein, is known to regulate the phenotypes of epithelial cells by modifying actin organization and cell motility. Recently, a microarray study showed that Tpbg is upregulated in Thy1 glomerulonephritis (Thy1 GN). We hypothesized that Tpbg regulates cytoskeletal rearrangement and modulates phenotypic alteration in podocytes under pathological conditions. Methods: We examined Tpbg expression in Thy $1 \mathrm{GN}$ and Tpbg function in mouse podocytes. Results: We demonstrated that Tpbg is upregulated in the injured podocytes of Thy $1 \mathrm{GN}$. In vitro, immunofluorescence studies revealed that Tpbg colocalized with the focal adhesion protein, vinculin, in parallel with stress fiber formation. This colocalization was observed even when actin filaments were depolymerized with cytochalasin D. Tpbg localization at focal adhesions was induced by dom-
\end{abstract}

inant-active RhoA and suppressed by the ROCK1 inhibitor $Y$-26732. In addition, transforming growth factor- $\beta$ increased Tpbg expression at focal adhesions concurrently with rearrangement of stress fibers. Stress fiber formation was suppressed in differentiated podocytes transfected with fulllength Tpbg. Furthermore, knockdown of Tpbg using small interfering RNA decreased podocyte motility. Conclusion: Our findings suggest a novel role of Tpbg in the phenotypic alteration of injured podocytes, and we accordingly propose a new mechanism of glomerular injury in glomerulonephritis.

Copyright $\odot 2010$ S. Karger AG, Basel

\section{Introduction}

Podocyte injury is a critical determinant of the development of glomerular damage in glomerulonephritis, although mechanisms are not well understood [1-4]. Thyl glomerulonephritis (Thyl GN), a widely used model of human mesangial proliferative glomerulonephritis, involves mesangial cell death, mesangiolysis and aneurysmal dilatation of intraglomerular capillaries. Dilated

\section{KARGER}

(C) 2010 S. Karger AG, Basel

Fax +41613061234

E-Mail karger@karger.ch

www.karger.com
Accessible online at: www.karger.com/ajn
Hideharu Abe, MD, PhD

3-18-15 Kuramoto-cho

Tokushima 770-8503 (Japan)

Tel. +81 88633 7184, Fax +81 886339245

E-Mail abeabe@clin.med.tokushima-u.ac.jp 
capillaries may induce podocyte damage via altered physical forces [5] or changes in glomerular basement membrane composition [4]. In human IgA nephropathy, podocyte injury is induced by mesangial cell-derived cytokines [6].

Campean et al. [7] reported that trophoblast glycoprotein (Tpbg) was upregulated in glomeruli by an RNA microarray study using the Thyl model. Tpbg is a $72-\mathrm{kDa}$ leucine-rich repeat transmembrane glycoprotein. In normal adult tissues, Tpbg expression is low and restricted to certain epithelia. In many human carcinomas, Tpbg expression is upregulated and strongly associated with metastasis [8-10]. In fibroblasts and epithelial cells, Tpbg has marked effects on the actin cytoskeleton, cell motility, and cell attachment $[11,12]$. In addition, Tpbg is associated with differentiation and epithelial-mesenchymal transition of mouse and human embryonic stem cells [13-15]. We hypothesized that Tpbg serves to link mesangial proliferation and podocyte injury in mesangio-proliferative GN, as modeled by Thyl GN.

\section{Materials and Methods}

\section{Animals}

Male Wistar rats (CLEA Japan, Tokyo, Japan) weighing 180 $200 \mathrm{~g}$ were used. Rats were housed under specific pathogen-free conditions. All animal experiments were performed in accordance with institutional guidelines, and the Review Board of Tokushima University granted ethical permission for this study.

\section{Induction of Experimental Mesangial Proliferative}

Glomerulonephritis (Thy1 GN), Urinalysis and Isolation of

Glomeruli

Thyl GN was induced by a single intravenous injection of mouse anti-rat Thy-1.1 monoclonal antibody ( $1 \mathrm{mg} / \mathrm{kg}$; Cedarlane Laboratories, Ont., Canada). These rats were sacrificed at day 3 , 6 , and $14(\mathrm{n}=5-8)$ after administration of anti-Thy-1.1 antibody.

Before sacrifice, the rats were individually housed in metabolic cages with free access to water for 24-hour urine collection. Urinary protein concentration was determined by the Bradford method (Bio-Rad, Oakland, Calif., USA). Negative controls were six age-matched rats injected with vehicle only. Glomeruli were isolated from the renal cortex of the rats by differential thieving, with a purity of $>90 \%$.

\section{RNA Isolation and PCR}

The total RNA was extracted from isolated rat glomeruli or cultured podocytes by TRIzol (Takara, Ootsu, Japan). The following PCR primers were used: rat Tpbg: sense $5^{\prime}$-GTCCTTCACAACTCCACCTTG-3', antisense 5'-CGGCACCACCTCTGTCTCTTTA-3', yielding a 135-bp PCR product; rat nephrin: sense $5^{\prime}$ TGAAGACACAGACCACCAGC-3', antisense 5'-GGAGAGCAGCAGAAGACCAC-3', yielding a 102-bp PCR product; rat WT1: sense 5'-TTGAAGGGAATGGCTGCTGG-3', antisense 5'-GAG-
GATGGGGGTTGTGTGG-3', yielding a 105-bp PCR product; rat transforming growth factor- $\beta$ (TGF- $\beta$ ): sense $5^{\prime}$-CTGACCCCCACTGATACG-3', antisense 5'-CACTGAAGCGAAAGCCCTG-3', yielding a 103 -bp PCR product; 18 SrRNA: sense $5^{\prime}$ GACTCAACACGGGAAACC-3', antisense 5'-CGGACATCTAAGGGCATCAC-3', yielding a 270-bp PCR product; murine Tpbg: sense 5'-CTACTGCTGCTTTGCTCACGC-3', antisense 5'-CACCTCCTCAACTCCTTTGTTG-3', yielding a 160-bp PCR product; murine GAPDH: sense 5'-AAAATGGTGAAGGTCGGTGTG-3', antisense 5'-AATGAAGGGGTCGTTGATGG-3', yielding a 110-bp PCR product).

PCR products were polymerized using cDNA solution and GoTaq (Promega, Madison, Wisc., USA). Quantitative PCR was performed on an ABI PRISM 7700 sequence detector (Applied Biosystems, Carlsbad, Calif., USA) using SYBR Green PCR Master Mix (Applied Biosystems) and specific primers following the manufacturer's protocols. For quantitative PCR of mouse Tpbg, a TaqMan probe of Tpbg and universal PCR Master Mix were used.

\section{Western Blot Analysis}

Isolated glomeruli and cultured podocytes were incubated in a lysis buffer (10 mM Tris, $150 \mathrm{mM} \mathrm{NaCl}, 1.0 \% \mathrm{NP}-40$, proteinase inhibitors) for $30 \mathrm{~min}$ at $4^{\circ} \mathrm{C}$. Protein concentrations were measured by DC protein assay (Bio-Rad Laboratories, Hercules, Calif., USA). Protein samples were heated to $100^{\circ} \mathrm{C}$ for $3 \mathrm{~min}$ in SDS gel-loading buffer, $20 \mu \mathrm{g}$ of each glomerular sample was applied to SDS gel electrophoresis and proteins were transferred to nitrocellulose filters (GE Healthcare, Little Chalfont, UK). The blots were incubated with anti-Tpbg antibody, followed by incubation with horseradish peroxidase-conjugated goat anti-rabbit IgG (Zymed, San Francisco, Calif., USA).

Tpbg Antibody

Polyclonal anti-Tpbg antibody was raised in a rabbit against peptides corresponding to carboxyl-terminal region of mouse Tpbg (INADPRLTNLSSNSDV), and the IgG fraction was purified using protein A sepharose. This peptide sequence corresponds to $93 \%$ of carboxyl-terminal region of rat Tpbg. This antibody recognizes specifically a band about $72 \mathrm{kDa}$ in rat glomerular lysate and in mouse podocyte lysate. Antibody specificity was confirmed by peptide blocking assay in in vivo immunostaining. Tpbg antibody was preabsorbed overnight with 25 times the concentration of Tpbg peptide.

\section{Histological Examination}

For light microscopy, tissues were fixed in methyl Carnoy's solution, and $2-\mu \mathrm{m}$ paraffin sections were stained with periodic acid-Schiff. Glomerulosclerosis score was semiquantitatively analyzed. The percentage of each glomerulus occupied by mesangial matrix was estimated and assigned a code as follows: $0=$ absent; $0.5=1-5 \% ; 1=5-25 \% ; 2=25-50 \% ; 3=50-75 \%$, or $4=75-100 \%$. The total number of cells in the glomeruli was counted in a blind protocol and computed for 20 full-sized glomeruli $(80-100 \mu \mathrm{m})$ for each kidney. For immunofluorescence microscopy, frozen $4-\mu \mathrm{m}$ sections were fixed in acetone for $10 \mathrm{~min}$ at $4^{\circ} \mathrm{C}$. For doublelabeled immunofluorescence microscopy for Tpbg with synaptopodin or nephrin, mouse monoclonal anti-synaptopodin antibody (Progen, Heidelberg, Germany), goat polyclonal anti-nephrin antibody (Santa Cruz, Santa Cruz, Calif., USA) and rabbit polyclonal anti-Tpbg antibody were used as primary antibody. 
Alexa Fluor 488- or Alexa Fluor 594-conjugated secondary antibodies (Invitrogen, Carlsbad, Calif., USA) were used. For double immunostaining for Tpbg with WT1, sections were incubated with rabbit polyclonal anti-WT1 antibody (Santa Cruz). Next, after blocking with the avidin solution and biotin solutions, sections were incubated with biotin-conjugated rabbit polyclonal anti-Tpbg antibody, followed by Texas Red-conjugated streptavidin (Zymed).

\section{Cell Culture}

Conditionally immortalized murine podocytes were provided by Dr. Peter Mundel. Podocytes were cultured in a RPMI-1640 (Sigma) medium containing 10\% fetal bovine serum, $100 \mathrm{U} / \mathrm{ml}$ penicillin, $0.1 \mathrm{mg} / \mathrm{ml}$ streptomycin and $2 \mathrm{mM} \mathrm{L}$-glutamine. For propagation, cells were cultivated with a culture medium supplemented with $50 \mathrm{U} / \mathrm{ml}$ of recombinant mouse $\gamma$-interferon (PeproTech, London, $\mathrm{UK}$ ) at $33^{\circ} \mathrm{C}$ with $5 \% \mathrm{CO}_{2}$ (permissive conditions). To induce differentiation, cells were cultured on a type I collagen at $37^{\circ} \mathrm{C}$ without $\gamma$-interferon for 14 days (nonpermissive conditions). Podocytes between passage 20 and 25 were used for all experiments.

\section{Cytokine and Inhibitors}

Differentiated podocytes were treated with $5 \mu \mathrm{M}$ cytochalasin D (Sigma) for $2 \mathrm{~h}$ to disrupt the actin cytoskeleton. Stress fibers were disrupted by treating cells with $10 \mu \mathrm{M}$ of the specific Rho kinase (ROCK) inhibitor Y-27632 (Wako, Osaka, Japan). To examine the effect of TGF- $\beta$ on Tpbg expression and actin filament, differentiated podocytes were treated with TGF- $\beta$ (PeproTech) for $24 \mathrm{~h}$, after serum starvation in 1\% RPMI. To inhibit the effect of TGF- $\beta$ type I receptor, the activin-like kinase receptor 5 (ALK5), podocytes were treated with $0,1.0$ and $10 \mu \mathrm{M}$ of the ALK5 inhibitor SB 431542 (Sigma).

\section{Immunocytochemistry and Confocal Laser Scanning}

Microscopy

For immunofluorescence, cells were fixed using $2 \%$ paraformaldehyde and permeabilized with $0.1 \%$ Triton X. Actin filaments were visualized with Texas Red-conjugated phalloidin (Invitrogen). Rabbit polyclonal anti-Tpbg antibody at $80 \mu \mathrm{g} / \mathrm{ml}$, mouse monoclonal anti-vinculin antibody (Sigma), mouse monoclonal anti-myc antibody (MBL, Nagano, Japan) and mouse monoclonal anti-FLAG antibody were used as primary antibody. Propidium iodide was used as nuclear staining. For peptide blocking assay, Tpbg antibody was preabsorbed overnight with 25 times the concentration of Tpbg peptide. Specimens were viewed with a confocal laser scanning microscopy (Leica, Wetzlar, Germany).

\section{Plasmids and Transfection}

Podocytes were transfected with Myc-tagged dominant active (pEFBOS-Myc-RhoA-Q14N) RhoA, dominant negative (pEFBOSMyc-RhoA-T18N) RhoA and full-length Tpbg constructs. Fulllength Tpbg cDNA were amplified by RT-PCR from podocyte RNA and inserted into $3 \times$ FLAG-CMV ${ }^{\mathrm{TM}}-14$ expression vector (Sigma). The authenticity of these expression plasmids was confirmed by DNA sequencing. Transient transfection of podocytes was performed using FuGene 6 reagent (Roche, Indianapolis, Ind., USA).

Tpbg and Podocyte Injury in Thyl GN
Immunofluorescence Intensity Quantification of Stress Fiber, Tpbg and Vinculin Signal

Areas and signal intensities of actin fibers, Tpbg and vinculin were identified by immunofluorescence. Positive areas were then converted to black pixels and traced, and the size and signal intensity were measured. The intensity of stress fibers, the ratio of stress fibers to total cell size, and the intensity of Tpbg and vinculin at the focal adhesion were quantified using Multi Gauge v2.2 software (Fujifilm, Tokyo, Japan).

\section{Small Interfering RNA Studies}

Small interfering RNA (siRNA) against Tpbg (si-Tpbg) and scrambled control siRNA (si- scramble) were purchased from Sigma. Three si-Tpbgs targeted to different sites in the message were used. Differentiated podocytes were transfected with siRNA using N-TER Nanoparticle siRNA transfection system (Sigma). To improve the transfection efficiency, repeated transfection of siRNA was performed at 0 and $24 \mathrm{~h}$. Tpbg expression in podocytes was analyzed by real-time PCR $48 \mathrm{~h}$ after first transfection.

\section{Wound Closure Assay}

Differentiated podocyte monolayer culture on coverslips was wounded with a $200-\mu l$ pipette tip after transfection with siscramble or si-Tpbg and incubated in the absence or presence of $10 \mathrm{ng} / \mathrm{ml}$ TGF- $\beta$ for $24 \mathrm{~h}$. Cell layers were stained with DAPI, photographed using BZ-8000 (Keyence, Osaka, Japan), and the wound width was measured. Migratory rates were calculated as (wound width $0 \mathrm{~h}$ - wound width $24 \mathrm{~h}$ )/wound width $0 \mathrm{~h} \times 100 \%$.

\section{Statistical Analysis}

Data are expressed as mean \pm SD. Statistical significance was defined as $\mathrm{p}<0.05$ and was evaluated using one-factor analysis of variance or Kruskal-Wallis test followed by a post-hoc test.

\section{Results}

\section{Tpbg RNA and Protein Were Expressed in Rat Glomeruli}

Expression of Tpbg mRNA was observed in rat isolated glomeruli by RT-PCR (fig. 1a). Specific reaction of the anti-Tpbg antibody with the $72-\mathrm{kDa}$ Tpbg was also demonstrated in glomerular whole lysate by Western blot analysis (fig. 1b).

\section{Expression of Tpbg Was Upregulated in the Injured \\ Podocytes of Thyl GN Rats}

We examined the expression of Tpbg in Thyl GN. Histological examination showed mesangiolysis at day 3 and the increase of mesangial matrix at day 6 . Significant increase in total cell count and extracellular matrix was observed at day 6 (fig. 2a). Urinary protein excretion significantly increased at both day 3 and day 6. Quantitative PCR showed that Tpbg expression in glomeruli significantly increased by $130 \%$ at day 6 ( $p<0.01$; fig. $2 c$ ). Glo- 
Fig. 1. Expression of Tpbg in rat glomeruli. a Expression of Tpbg mRNA in rat glomeruli. RT-PCR yielded a 135-bp product for Tpbg. NC denotes negative control with glomerular mRNA and without reverse transcription. G denotes glomerular sample. b Expression of Tpbg protein in rat glomeruli. Glomerular whole lysate was subjected to immunoblot analysis with an anti-Tpbg antibody.

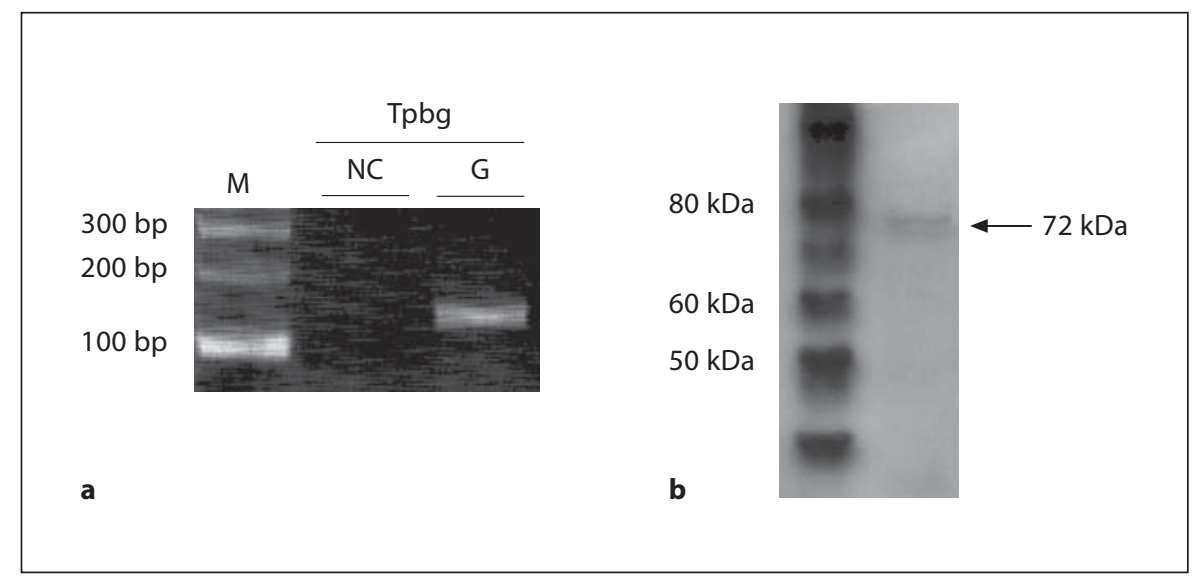

merular expression of Tpbg protein also significantly increased at day 6 compared with control (fig. 2d). By double immunofluorescence of control rat glomeruli, Tpbg was faintly stained and partially colocalized with nephrin (fig. 2e). Tpbg was stained in cell bodies of podocyte labeled with WT1 at day 6 (fig. 2e). Tpbg partially colocalized with synaptopodin (fig. $2 \mathrm{~h}$ ) or nephrin (fig. 2i), perhaps due to expression in the central portions of podocytes. At day 6 , no positive staining was detected with control IgG (fig. 2e) and Tpbg antibody preabsorbed with the peptide that was used for immunization (online suppl. fig. 1; for all online supplementary material, see www. karger.com/doi/10.1159/000321366).

Quantitative PCR showed that both nephrin and WT1 significantly decreased concomitantly with mesangial cell proliferation (fig. 2j). These results indicate that Tpbg expression is upregulated in podocytes damaged in Thyl GN. We hypothesized that TGF- $\beta$ mediates podocyte injury in glomerulonephritis. Quantitative PCR showed that TGF- $\beta$ expression began to increase at day 3 and peaked at day 6 (fig. $2 \mathrm{k}$ ).

\section{Tpbg Was Localized to Focal Adhesions along with}

Cytoskeletal Remodeling in Differentiated Podocytes

To investigate the function of Tpbg in vitro, we used conditionally immortalized murine podocytes. Quantitative PCR showed that mRNA expression in differentiated cells was significantly decreased compared with undifferentiated cells (fig. 3a). By immunoblot analysis, we detected a $72-\mathrm{kDa}$ protein (Tpbg) in the whole lysate of both differentiated and undifferentiated cells. Protein expression also significantly decreased in differentiated cells (fig. 3b).
Fig. 2. Upregulation of Tpbg expression in glomeruli in Thyl GN. a Representative light microscopic pictures of glomeruli (periodic acid-Schiff staining), total cell number and glomerulosclerosis score in Thyl glomerulonephritis. Significant increase in total cell number and extracellular matrix was observed at day 6 $(\mathrm{p}<0.01)$. Scale bars $=20 \mu \mathrm{m}$. $\mathbf{b}$ Time course of proteinuria in Thy1 glomerulonephritis. Data are expressed as mean \pm SD. Urinary protein excretion significantly increased at both day 3 and day 6 ( $\mathrm{n}=5-8$ in each group, $\mathrm{p}<0.01)$. $\mathbf{c}$ Expression of Tpbg mRNA in glomeruli from Thyl glomerulonephritis. Quantitative evaluation of Tpbg mRNA was performed by real-time PCR. Expression of Tpbg mRNA was significantly increased, paralleling the mesangial proliferative changes (day $6, \mathrm{p}<0.01$ ). 18SrRNA was used as internal control. The data are expressed as mean $\pm S D$, with $n=5-6$ in each group. $\mathbf{d}$ Expression of Tpbg protein in glomeruli from Thyl glomerulonephritis. Glomerular lysates were subjected to immunoblot analyses with an anti-Tpbg antibody. Representative bands from two to three independent experiments are shown. Tpbg was significantly upregulated at day 6 in the course of Thyl glomerulonephritis $(n=5-6 ; p<0.01)$. e Double immunofluorescence for Tpbg (Alexa Fluor 488) with nephrin (Alexa Fluor 594) in control rat glomeruli. Tpbg was faintly stained and partially colocalized with nephrin. $\mathbf{f}-\mathbf{i}$ Double immunofluorescence for Tpbg (Alexa Fluor 594 or 488) or control IgG with WT1 (Alexa Fluor 488), with synaptopodin (Alexa Fluor 488) or nephrin (Alexa Fluor 594) in rat glomeruli at day 6. Tpbg was expressed in WT1-positive cells (podocytes; g) and partially colocalized with synaptopodin (h) and nephrin (i). Segmental decrease in staining for synaptopodin, a marker for podocyte foot processes, was observed, suggesting podocyte injury (论). e No positive staining was detected with control IgG. Scale bars $=20 \mu \mathrm{m}$. $\mathbf{j}$ Expression of nephrin and WT1 mRNA in Thyl GN. Quantitative evaluation of nephrin and WT1 mRNA was performed by real-time PCR. 18SrRNA was used as an internal control. Expression of nephrin and WT1 mRNA was significantly decreased. Day 6 versus control, ${ }^{*} \mathrm{p}<0.01$; day 14 versus control, ${ }^{* *} \mathrm{p}<0.05 . \mathbf{k}$ Expression of TGF- $\beta$ mRNA in glomeruli from Thyl GN. Quantitative evaluation of TGF- $\beta$ mRNA was performed by real-time PCR. 18SrRNA was used as an internal control. Expression of TGF- $\beta$ mRNA was significantly increased (day 6, p < 0.05). 

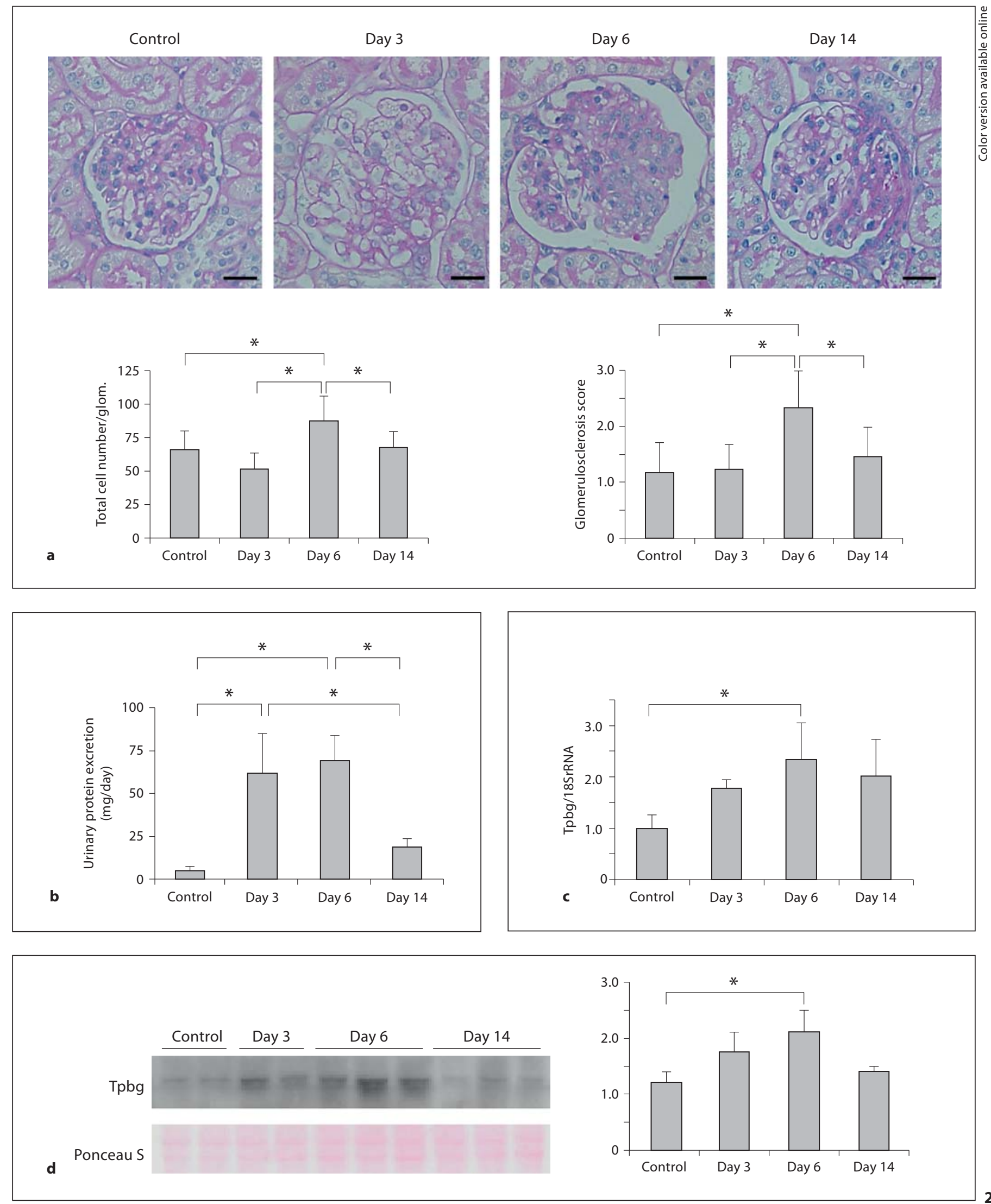

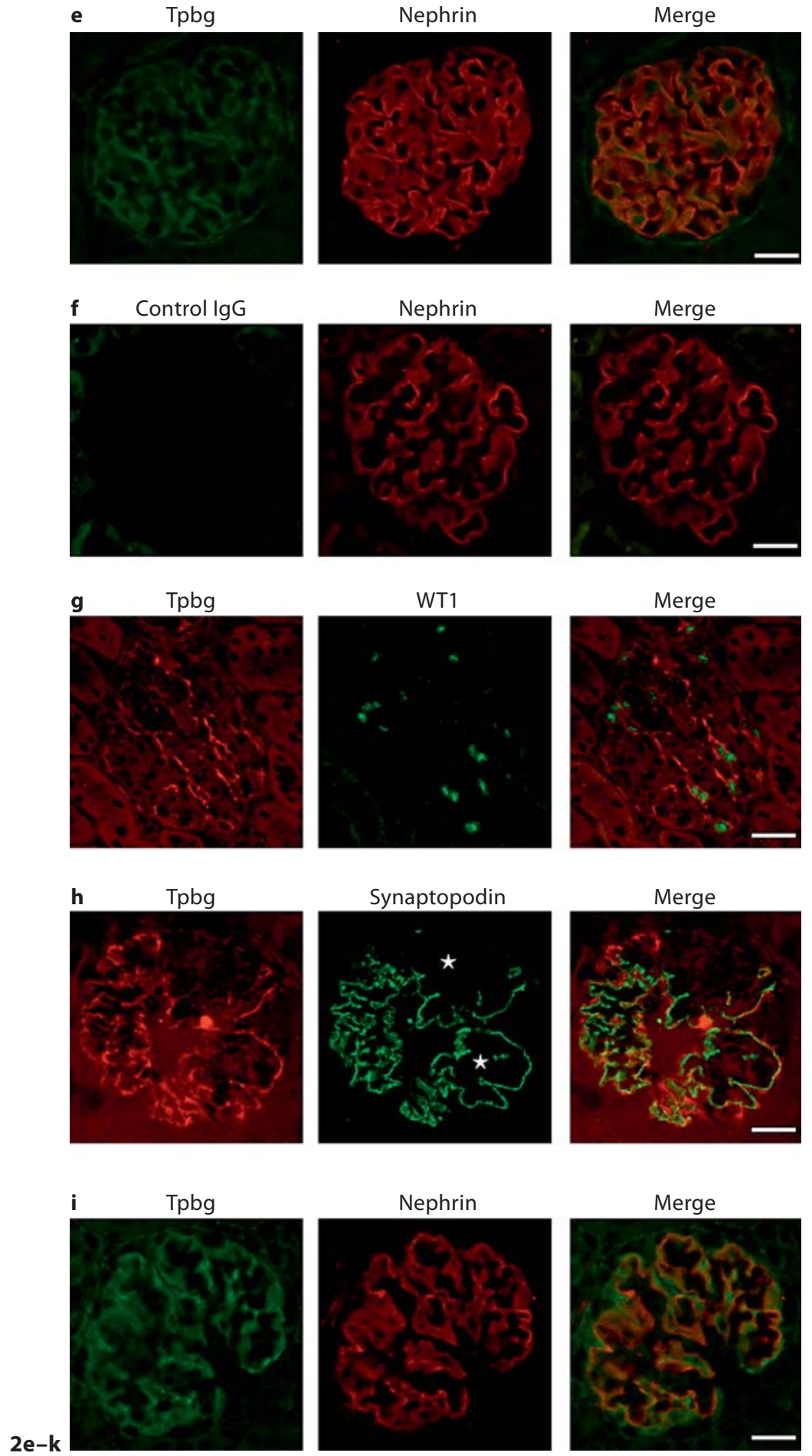
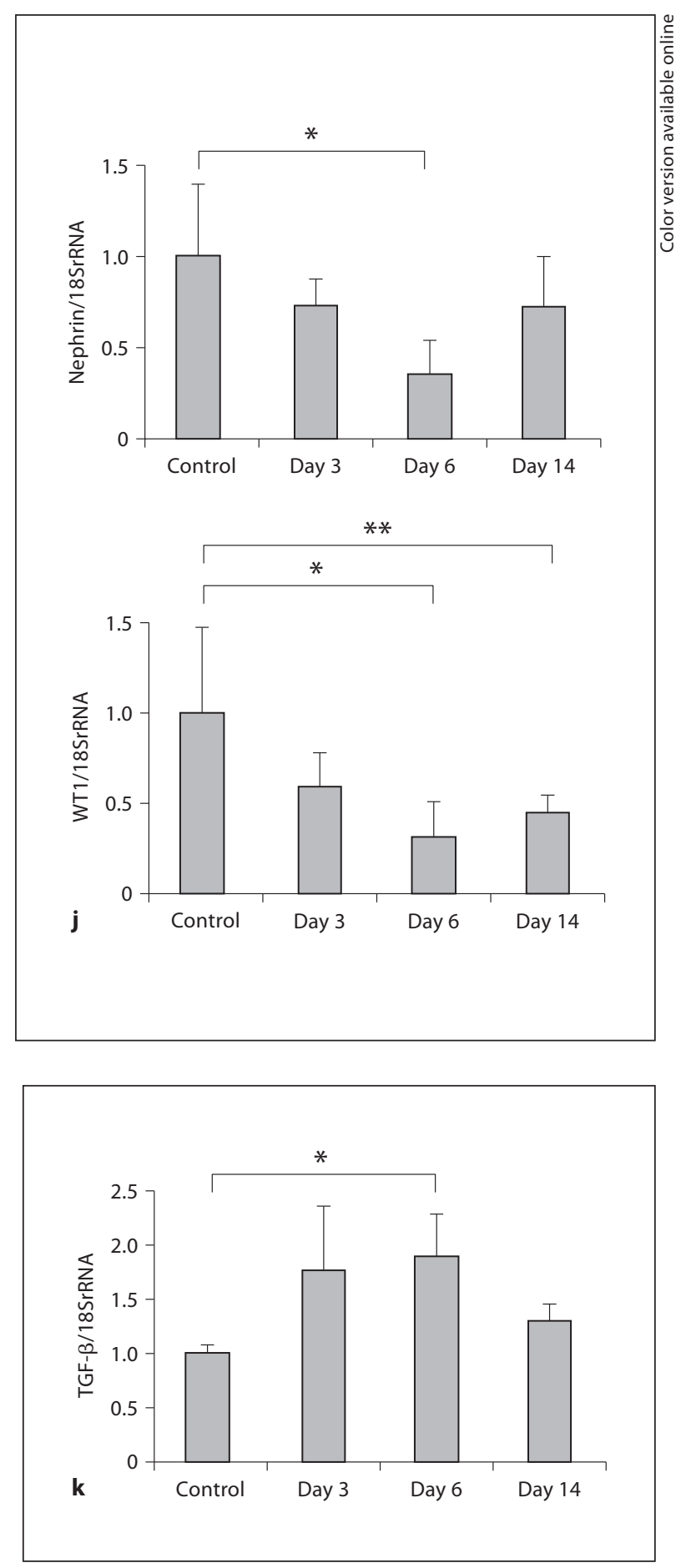
Fig. 3. Localization of Tpbg at focal adhesions along with cytoskeletal remodeling in murine podocytes. a Expression of Tpbg mRNA in undifferentiated and differentiated cultured podocytes. Gene expression was determined by RT-PCR and DNA sequence. RT-PCR yielded a 159-bp product for mouse Tpbg. NC denotes negative control with podocyte mRNA and without reverse transcription, $S$ denotes reverse transcription sample. Quantitative PCR showed that mRNA expression in differentiated cells was significantly decreased compared with undifferentiated cells ( $\mathrm{n}=$ 3 ; $\mathrm{p}<0.01$ ). b Whole lysates of undifferentiated and differentiated cultured podocytes were subjected to immunoblot analyses with an anti-Tpbg antibody. In both lanes, $72-\mathrm{kDa}$ protein Tpbg was labeled in whole cell lysate. $\beta$-Actin was used as a protein loading control. Protein expression in differentiated cells was significantly decreased compared with undifferentiated cells ( $n=3$; $p<0.01)$. c-g Double immunofluorescence for Tpbg with F-actin, vinculin and propidium iodide. c Tpbg (Alexa Fluor 488) colocalized with actin (Texas Red phalloidin) at the cell margins of lamellipodia. d Stress fibers (Texas Red phalloidin) were induced, and Tpbg (Alexa Fluor 488) was localized to the tip of stress fibers in differentiated podocytes. e Higher magnification of boxed region in $\mathbf{d}$. f Tpbg (Alexa Fluor 488) partially colocalized with the focal adhesion protein vinculin (Alexa Fluor 594). a

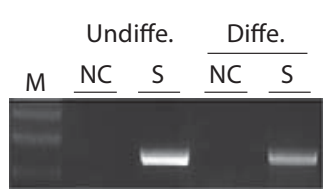

Undiffe. Diffe.

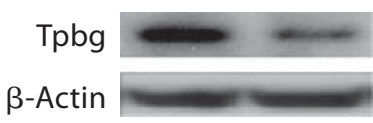

b
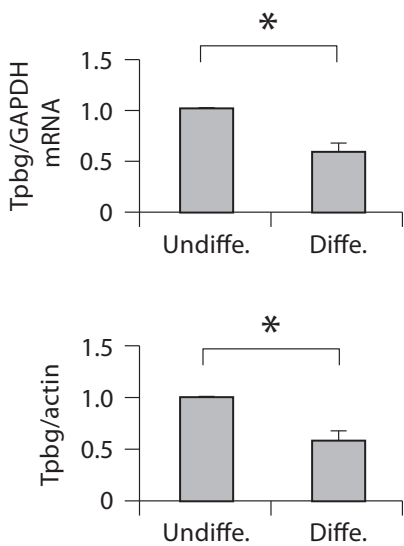

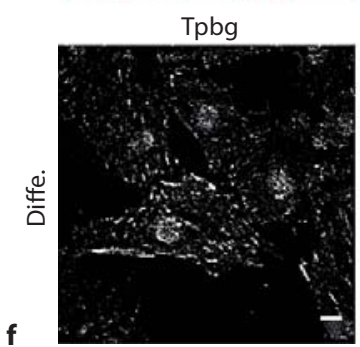

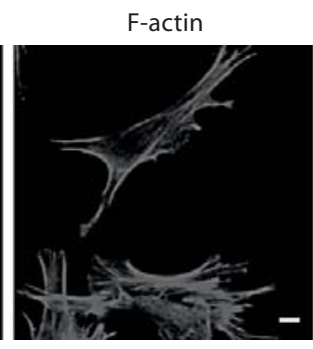

F-actin

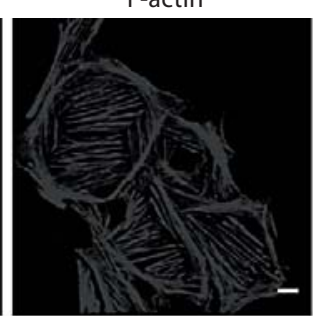

F-actin

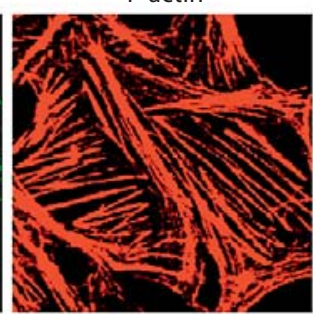

Vinculin

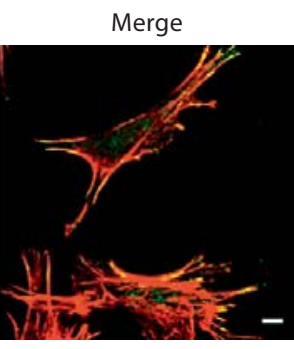

Merge

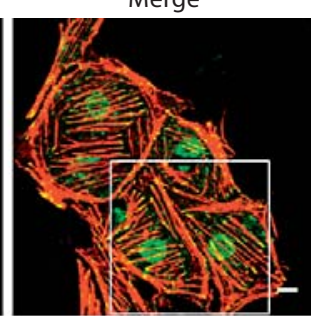

Merge

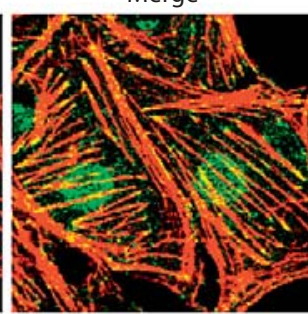

Merge
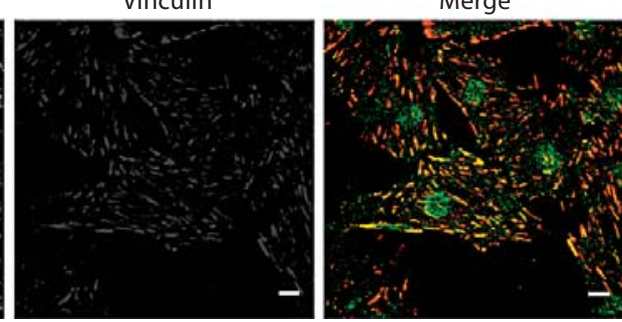
Fig. 3. Localization of Tpbg at focal adhesions along with cytoskeletal remodeling in murine podocytes. g Nuclei (propidium iodide) were also stained with Tpbg antibody labeled with Alexa Fluor 488. h Excess Tpbg-blocking peptide abolished the cytoplasmic staining, but did not completely block the nuclear staining (arrows). i In the presence of cytochalasin D, which inhibits actin polymerization, Tpbg (Alexa Fluor 488) still colocalized with short Factin filaments (Texas Red phalloidin) and with vinculin (Alexa Fluor 594) in differentiated podocytes. Scale bars $=10 \mu \mathrm{m}$.

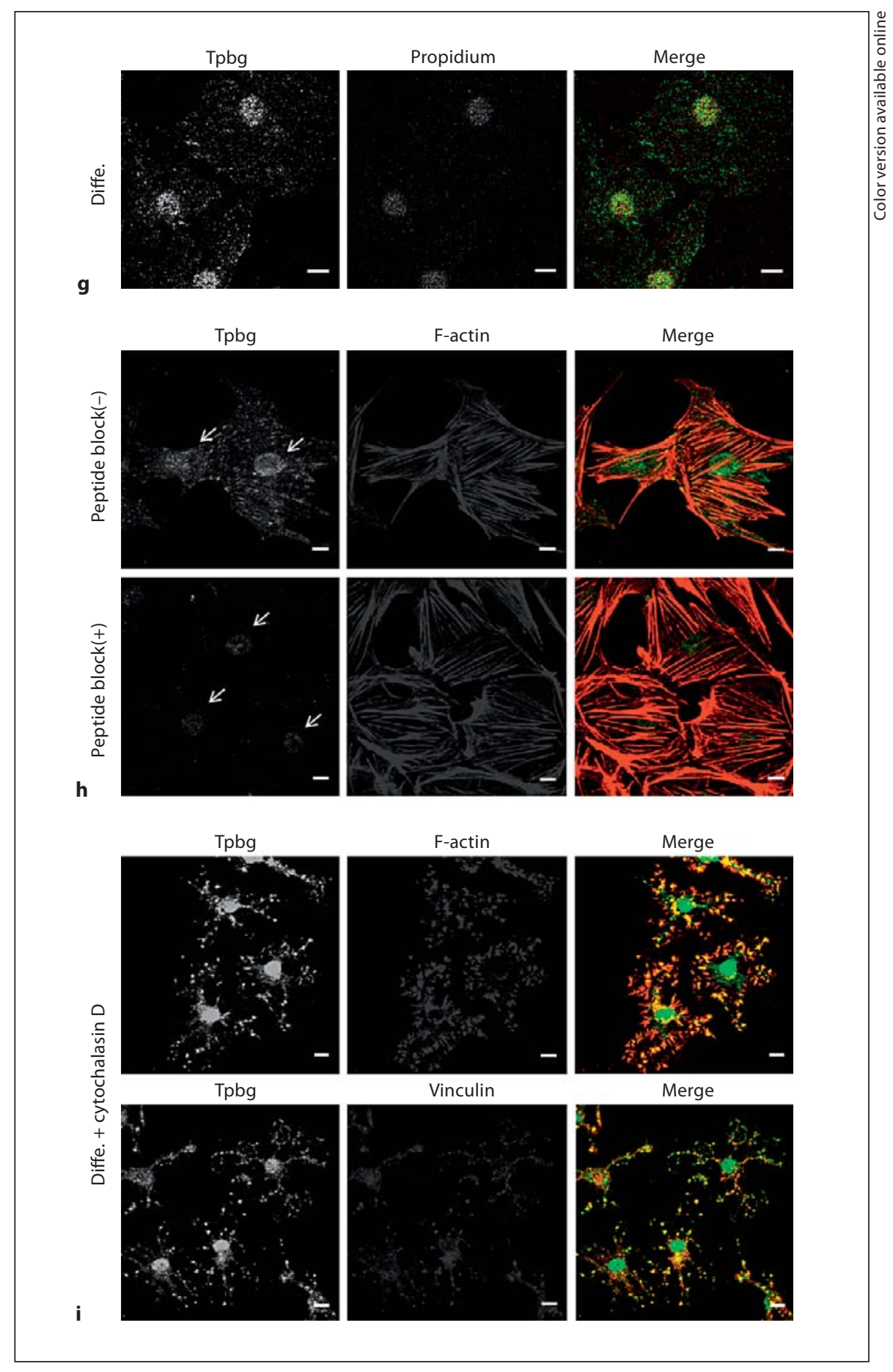


Fig. 4. Stress fiber formation induced by RhoA in undifferentiated podocytes. a-c Double immunofluorescence for Myc and F-actin in undifferentiated podocytes transfected with Myc empty vector, with DN-RhoA, or with DA-RhoA. Neither Myc empty vector (a) nor DN-RhoA (b) induced stress fibers. c DA-RhoA induced stress fibers in undifferentiated podocytes. Scale bars $=10 \mu \mathrm{m}$.

Tpbg and Podocyte Injury in Thyl GN

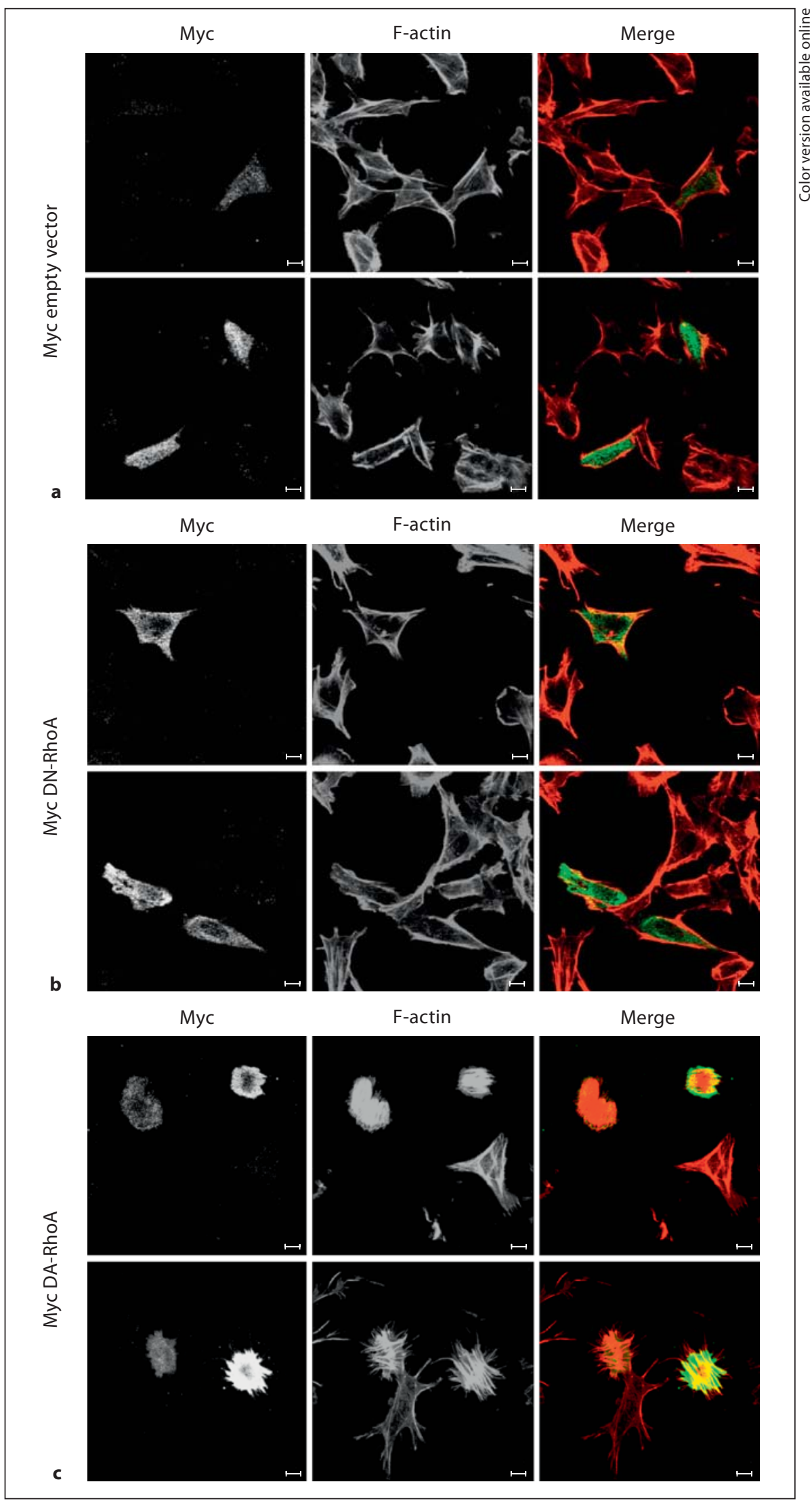

Am J Nephrol 2010;32:505-521 
Fig. 5. Tpbg localization at focal adhesions induced by RhoA in undifferentiated podocytes. a Double immunofluorescence for vinculin (Alexa Fluor 488) with F-actin (Texas Red phalloidin) in undifferentiated podocytes transfected with DA-RhoA. Vinculin localized to the tip of stress fibers induced by DA-RhoA, indicating focal adhesions. b Double immunofluorescence for Tpbg (Alexa Fluor 488) with F-actin in undifferentiated podocytes transfected with DA-RhoA. Tpbg localized to the tip of stress fibers induced by DA-RhoA. c Double immunofluorescence for Tpbg with vinculin in undifferentiated podocytes transfected with DA-RhoA. Tpbg colocalized with vinculin in undifferentiated podocytes transfected with DA-RhoA. Scale bars $=10 \mu \mathrm{m}$.

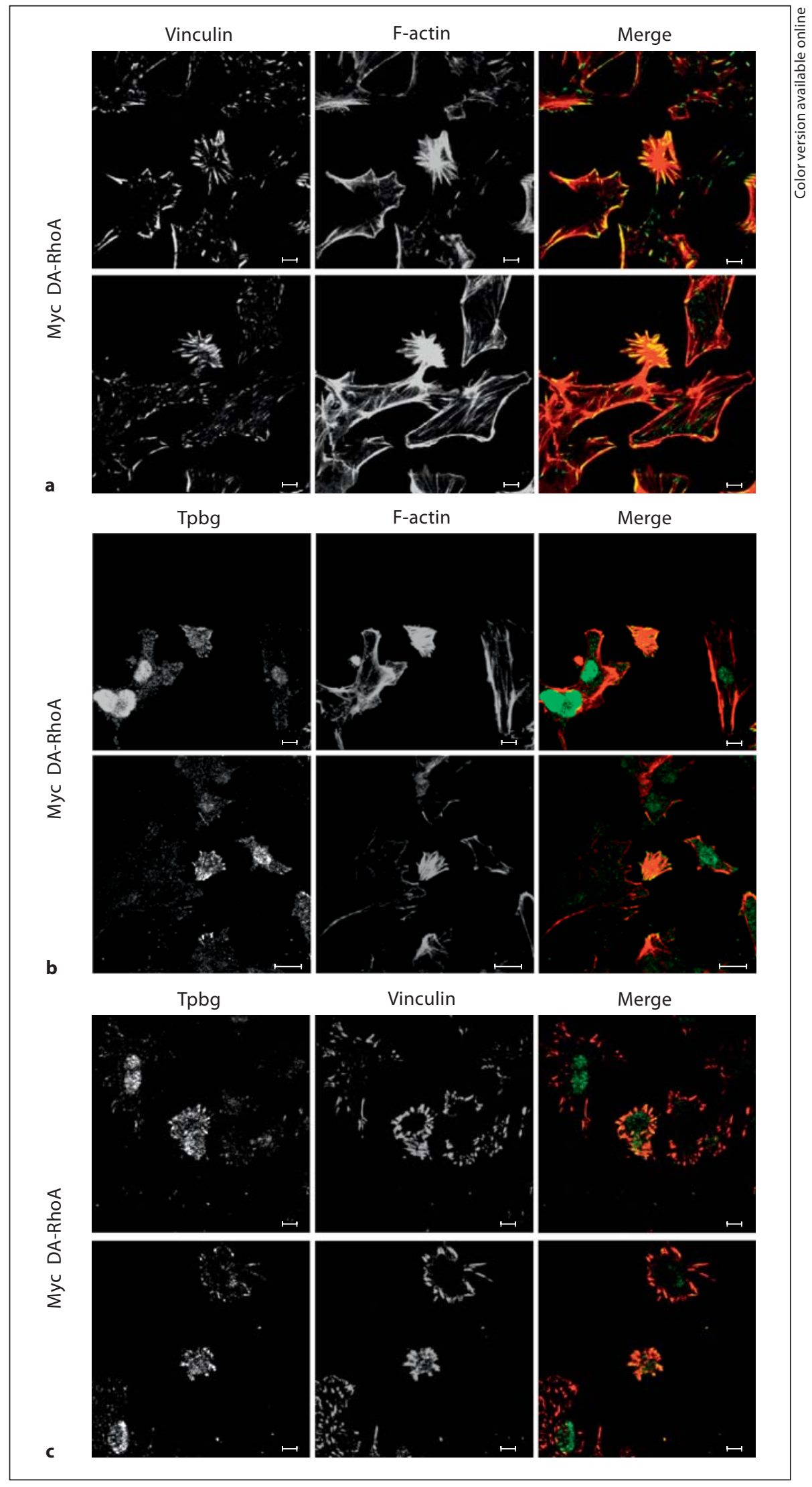


Fig. 6. Tpbg localization at focal adhesions in parallel with dynamic actin organization regulated by RhoA-ROCK signal in mouse podocytes. Double immunofluorescence for Tpbg (Alexa Fluor 488) and Factin (Texas Red phalloidin) or vinculin (Alexa Fluor 594) in differentiated podocytes in the absence or presence of the Rho inhibitor Y-27632. Differentiated podocytes were treated with 0 or $10 \mu \mathrm{M}$ Y-27632 for $12 \mathrm{~h}$. Y-27632 inhibited the formation of stress fiber and focal adhesion, and altered Tpbg distribution from being punctate, oriented in linear patterns, to diffuse punctuate staining. Washout of Y-27632 was associated with reformation of stress fiber and focal adhesion and restoration of Tpbg staining to the oriented pattern. Scale bars $=10 \mu \mathrm{m}$.

Tpbg and Podocyte Injury in Thyl GN

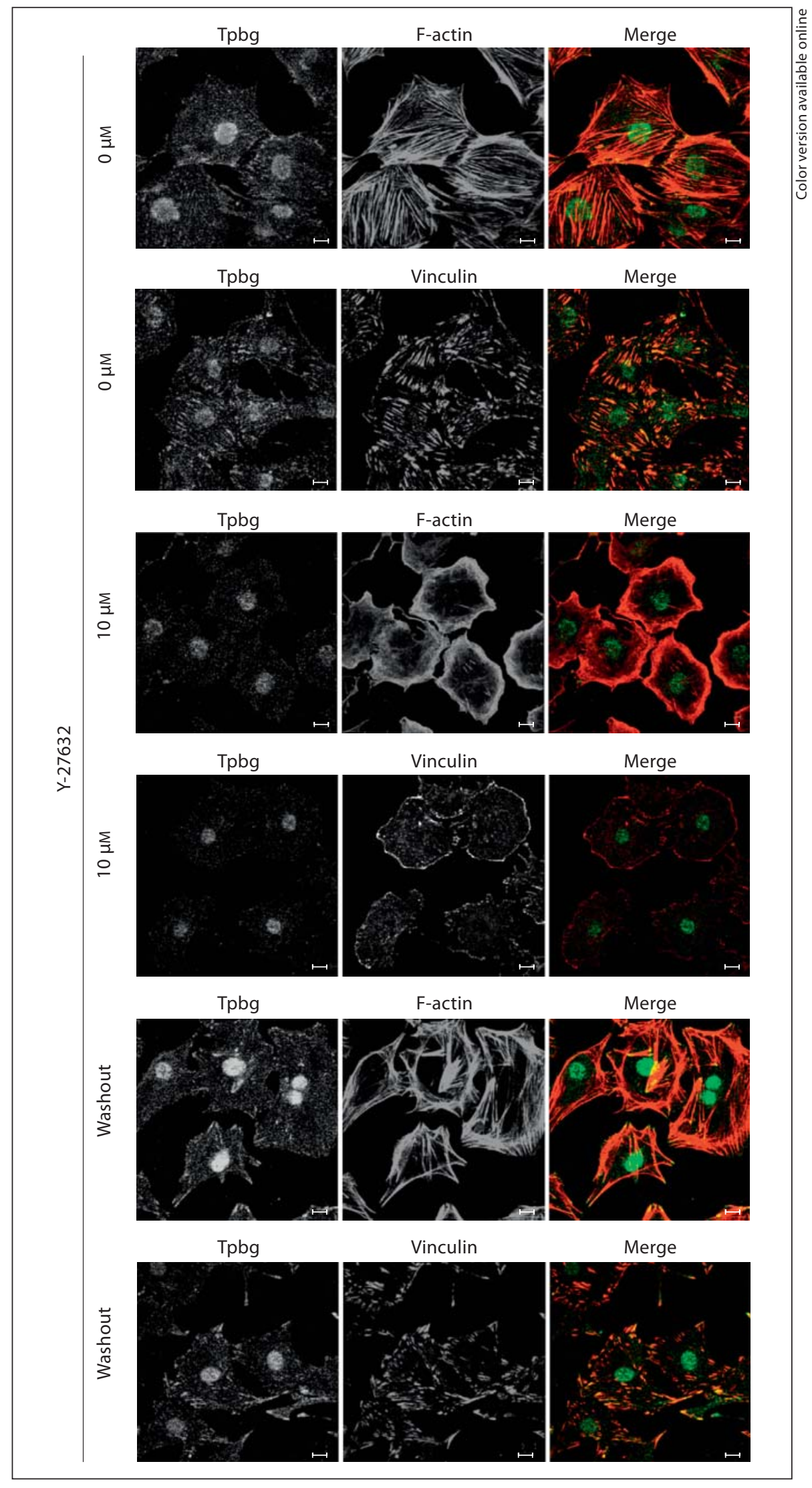

Am J Nephrol 2010;32:505-521 

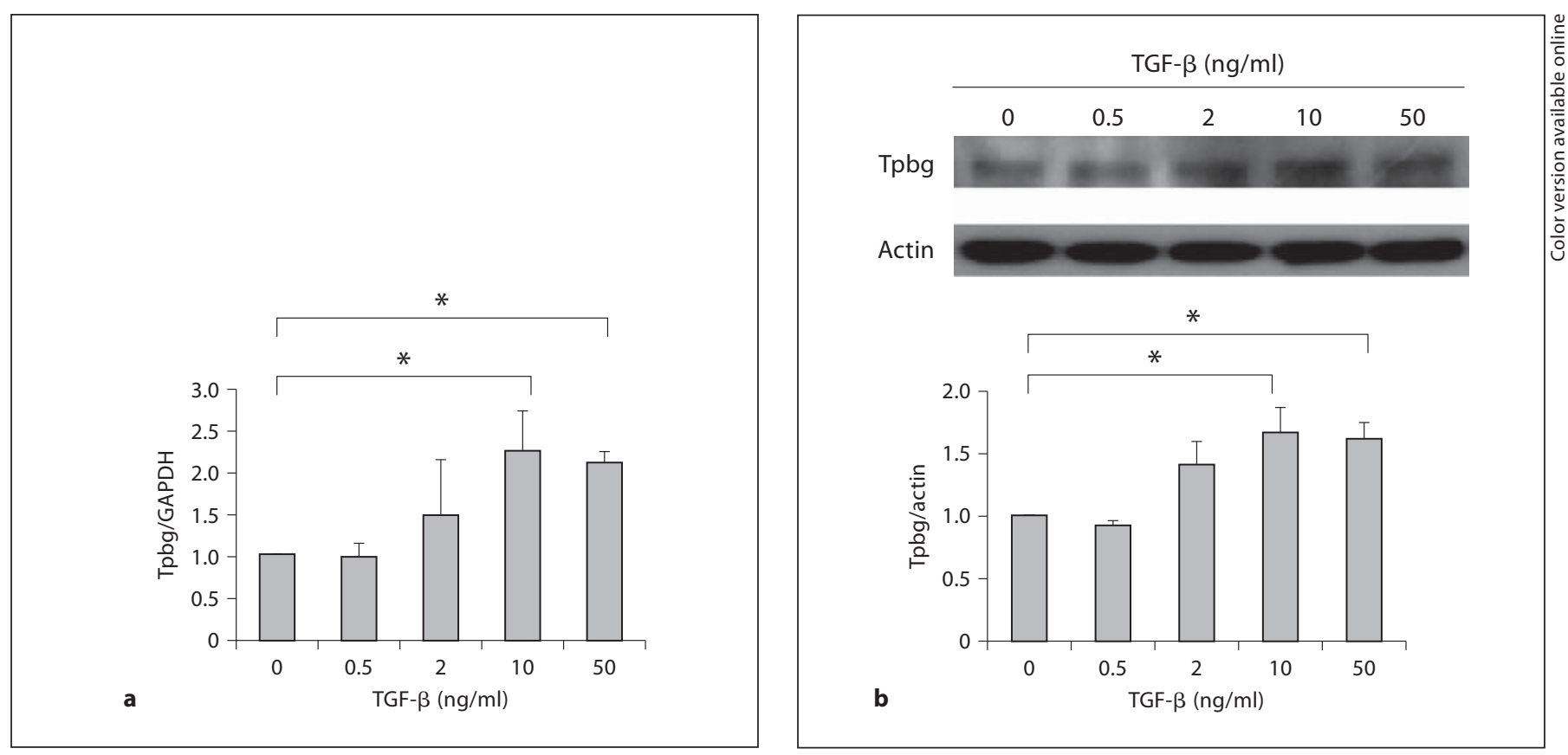

Fig. 7. Rearrangement of stress fibers and increased Tpbg localization at the focal adhesions by TGF- $\beta$ in cultured podocytes. a Expression of Tpbg mRNA in differentiated podocytes exposed to TGF- $\beta$. Real-time PCR showed that TGF- $\beta$ exposure for $24 \mathrm{~h}$ increased Tpbg mRNA expression in a dose-dependent manner $(\mathrm{n}=4 ; \mathrm{p}<0.01)$. GAPDH was used as an internal control. $\mathbf{b}$ Expression of Tpbg protein in differentiated podocytes exposed to TGF- $\beta$. Western blot analysis showed that TGF- $\beta$ exposure for $24 \mathrm{~h}$ significantly increased Tpbg protein expression in a dose-dependent manner $(\mathrm{n}=3$; $\mathrm{p}<0.01)$. $\beta$-Actin was used as an internal control. c Double immunofluorescence for Tpbg with F-actin (Texas Red phalloidin) and with vinculin (Alexa Fluor 594) in differentiated podocytes treated with TGF- $\beta$ for $24 \mathrm{~h}$. The number and intensity of cortical actin bundles with Tpbg localization at focal adhesions were increased in a dose-dependent manner. Scale bars $=10 \mu \mathrm{m}$.

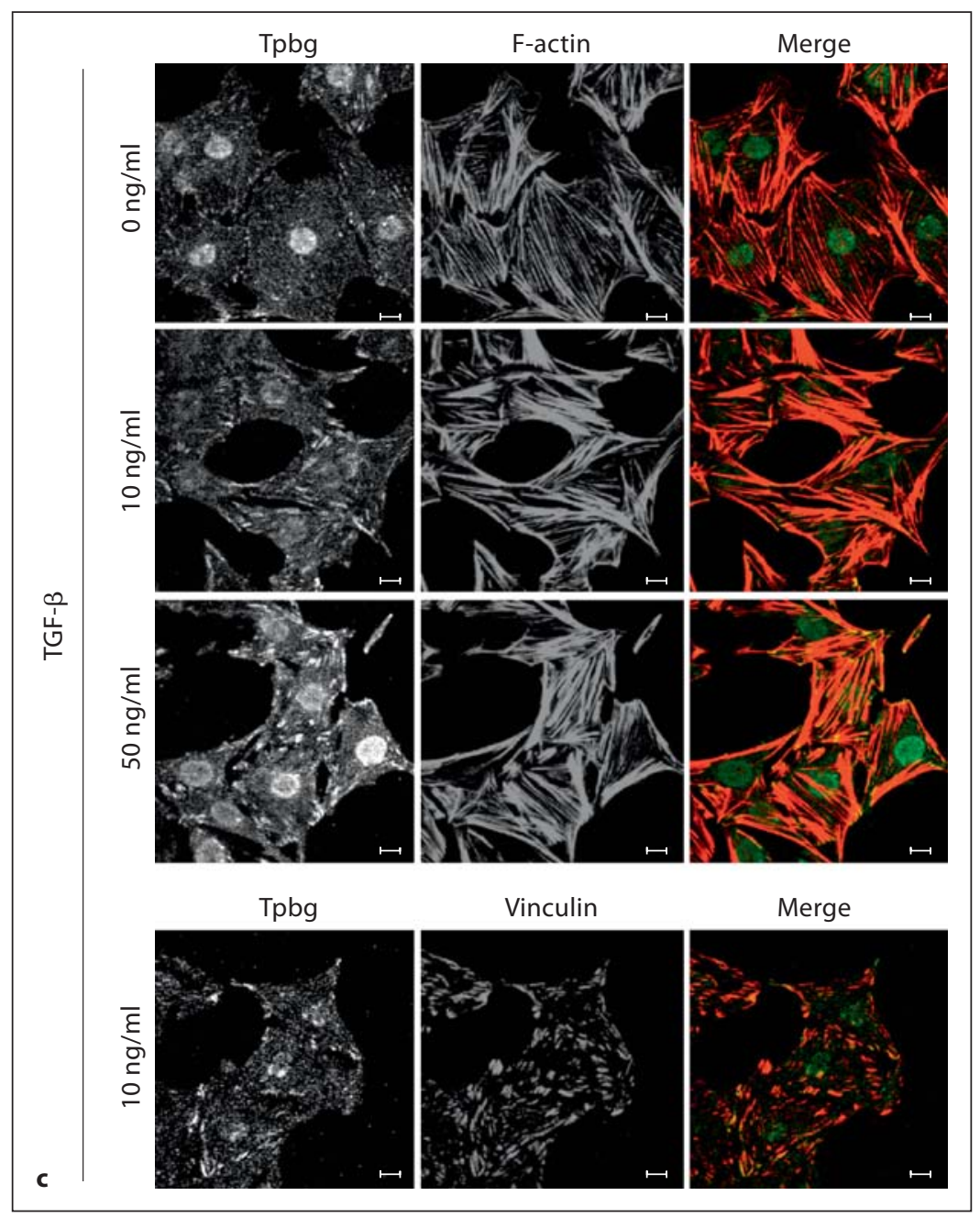



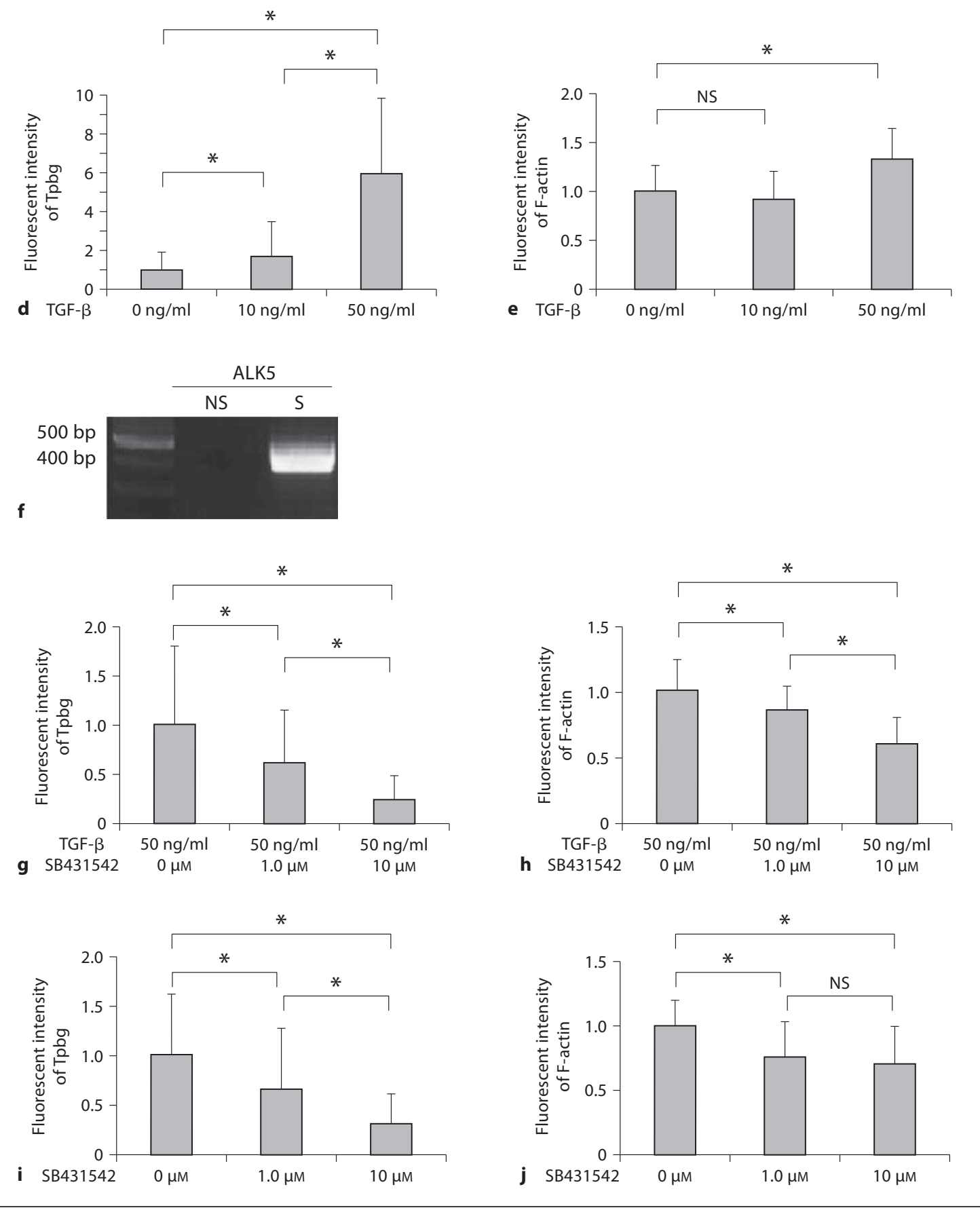

Fig. 7. Rearrangement of stress fibers and increased Tpbg localization at the focal adhesions by TGF- $\beta$ in cultured podocytes. d-e Statistical evaluation for fluorescent intensity of Tpbg and F-actin in differentiated podocytes treated with TGF- $\beta$. The intensity of Tpbg at focal adhesions (d) and F-actin (e) was significantly increased $(\mathrm{p}<0.01 ; \mathrm{n}=4)$. $\mathrm{f}$ Expression of ALK5 mRNA in cultured podocytes. RT-PCR yielded a 497-bp product for ALK5. NC denotes negative control without reverse transcription, $\mathrm{S}$ denotes reverse transcription sample. $\mathbf{g}, \mathbf{h}$ Quantitative evaluation for fluorescence intensity of Tpbg and F-actin in dif- ferentiated podocytes treated with SB431542 and TGF- $\beta$. Differentiated podocytes, pretreated with $0,1.0$, and $10 \mu \mathrm{M}$ SB431542, were stimulated with $50 \mathrm{ng} / \mathrm{ml}$ TGF- $\beta$ for $24 \mathrm{~h}$. The intensity of Tpbg at focal adhesions (g) and F-actin (h) was significantly decreased $(\mathrm{p}<0.01)$ by SB431542 $(\mathrm{n}=3)$. $\mathbf{i}-\mathbf{j}$ Quantitative evaluation of fluorescent intensity of Tpbg and F-actin in differentiated podocytes treated with SB431542. Differentiated podocytes were treated with SB431542 for $24 \mathrm{~h}$. The intensity of Tpbg at focal adhesion (i) and F-actin (j) was significantly decreased $(\mathrm{p}<0.01$, $\mathrm{n}=3$ ). 

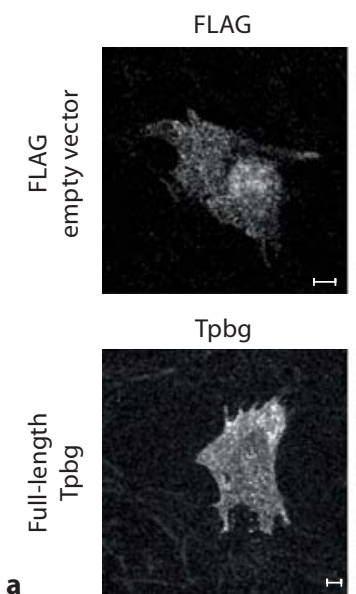

F-actin

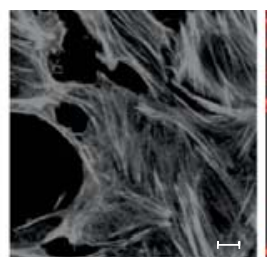

F-actin

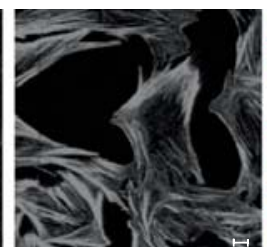

Merge

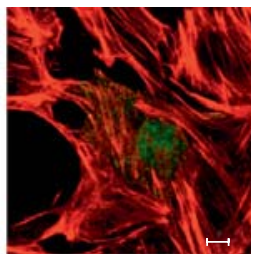

Merge

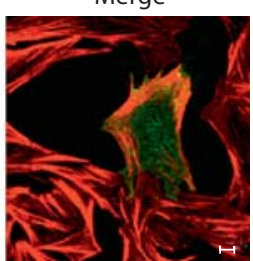

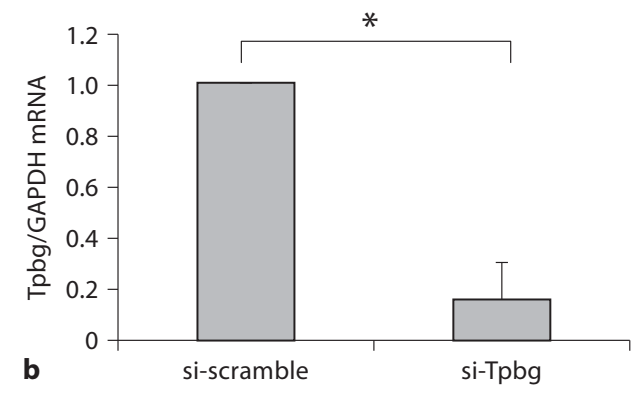

Fig. 8. Regulation of podocyte migration and actin cytoskeleton by Tpbg. a Double immunofluorescence for F-actin (Texas Red phalloidin) with FLAG (Alexa Fluor 488) and Tpbg (Alexa Fluor 488) in differentiated podocytes transfected with FLAG empty vector or full-length Tpbg, respectively. In differentiated podocytes transfected with FLAG empty vector, stress fibers were maintained. Stress fibers were not observed in differentiated podocytes transfected with full-length Tpbg, while cortical actin was preserved as shown in the podocyte in the center of the field. Scale bars $=10 \mu \mathrm{m}$. b Expression of Tpbg mRNA in differentiated podocytes transfected with si-scramble or si-Tpbg. Expression of Tpbg mRNA was significantly reduced in podocytes transfected with siTpbg, compared with that in podocytes transfected with si-scramble $(\mathrm{p}<0.01)$. Data represent mean \pm SD of three independent experiments. c Wound closure assay. In podocytes transfected with siscramble or si-Tpbg, no significant difference of wound closure rate at $24 \mathrm{~h}$ was observed in the absence of TGF- $\beta$. Compared with control podocytes, Tpbg knockdown podocytes demonstrated significantly impaired cell migration in the presence of TGF- $\beta$ ( $p<0.01)$. A and B in panels reflect the width of the wound at 0 and $24 \mathrm{~h}$, respectively. Migratory rates were calculated as $(\mathrm{A}-\mathrm{B}) / \mathrm{A} \times 100 \%$. Data represent mean $\pm \mathrm{SD}$ of five independent experiments. Scale bars $=100 \mu \mathrm{m}$.

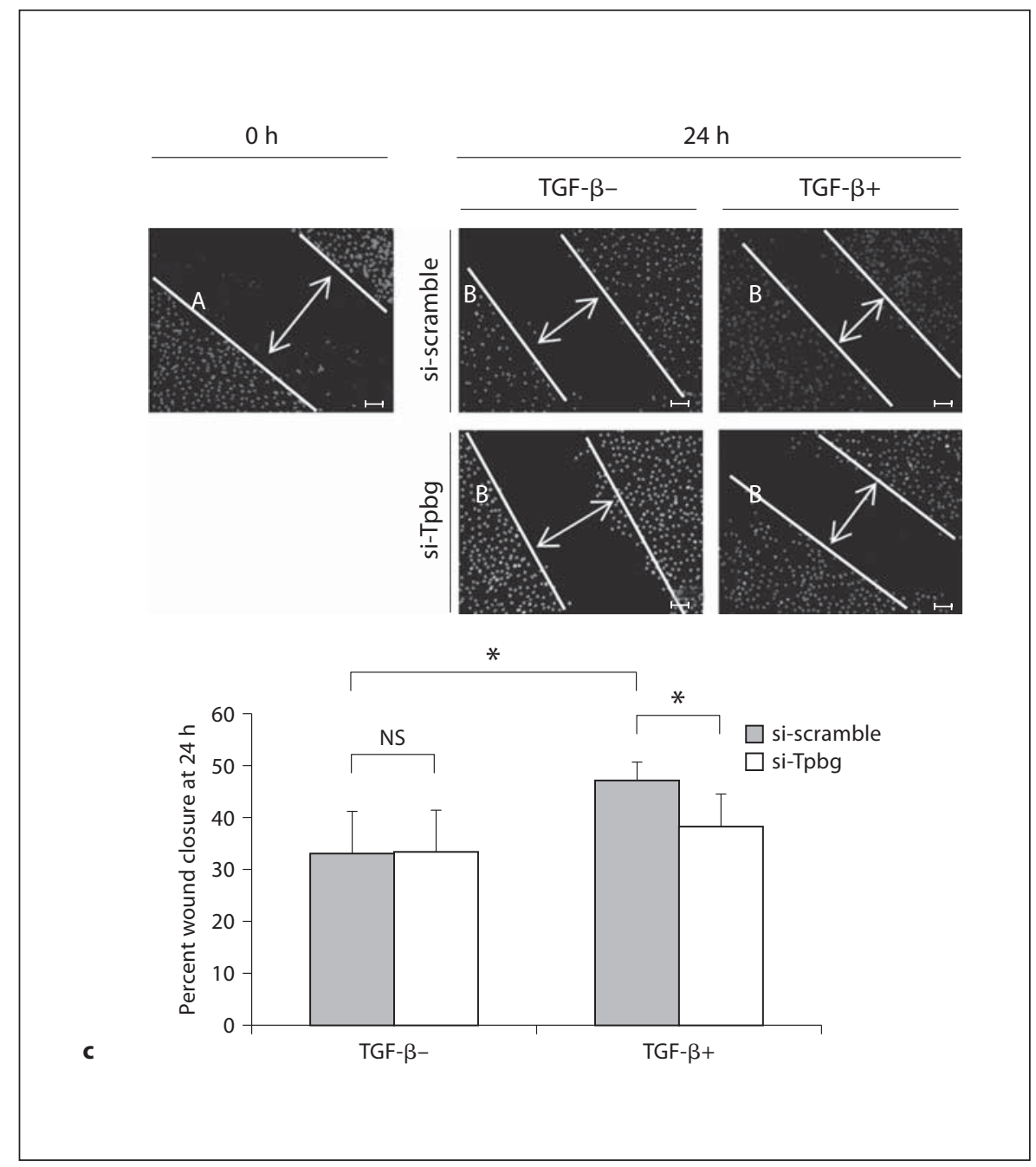


Immunofluorescence microscopy failed to detect any stress fibers in undifferentiated cells, but Tpbg colocalized with actin in the cell margins of lamellipodia (fig. 3c). In differentiated cells, stress fibers were present and Tpbg was localized to the tips of stress fibers (fig. $3 \mathrm{~d}, \mathrm{e}$ ).

We performed colocalization experiments for Tpbg with vinculin, which links integrins to the actin cytoskeleton at focal adhesions; we observed considerable colocalization of Tpbg and vinculin (fig. 3f). Nuclear staining with propidium iodide demonstrated that nuclei were also labeled with Tpbg antibody (fig. 3g). An excess of Tpbg-blocking peptide was able to abolish the cytoplasmic staining, but did not completely block the nuclear staining (fig. $3 \mathrm{~h}$, arrows); this result suggests that the cytoplasmic staining is specific and that the nuclear staining might be not specific.

We also investigated whether Tpbg localization requires stress fibers. In the presence of cytochalasin D, which inhibits actin polymerization, Tpbg still colocalized with short F-actin filaments and with vinculin (fig. 3i). This experiment suggests that stress fiber formation is not required for Tpbg localization to focal adhesions.

\section{Tpbg Localizes at Focal Adhesions in Parallel \\ with Dynamic Actin Organization Regulated by \\ RhoA-ROCK Signal in Mouse Podocytes}

RhoA and ROCK1, a downstream effector of RhoA, play prominent roles in regulating actin organization and focal adhesions in podocytes. In transient transfection studies, dominant active-RhoA (DA-RhoA) induced stress fibers (fig. 4c), while the negative controls Myc empty vector (fig. 4a) and the dominant negative-RhoA (DN-RhoA; fig. 4b) did not induce stress fibers. Further, in undifferentiated podocytes, DA-RhoA induced the formation of focal adhesions containing vinculin and Tpbg (fig. 5).

In the presence of ROCK1 inhibitor Y-27632 $(10 \mu \mathrm{M})$, differentiated podocytes lost stress fibers concomitantly with the decrease in Tpbg localization at focal adhesions. The re-formation of stress fibers after removal of Y-27632 was also accompanied by Tpbg localization at focal adhesions (fig. 6). These results demonstrate that Tpbg localizes to focal adhesions in parallel with the formation of stress fibers and focal adhesions regulated by RhoA.

TGF- $\beta$ Promoted Tpbg Expression and Localization at Focal Adhesions, Concurrently with Stress Fiber Rearrangement

TGF- $\beta$ is involved in regulation of the actin cytoskeleton in epithelial cells $[16,17]$. Following TGF- $\beta$ treat- ment for $24 \mathrm{~h}$, expression of Tpbg mRNA (fig. 7a) and protein (fig. $7 \mathrm{~b}$ ) was increased in a dose-dependent manner.

Treatment of differentiated podocytes with TGF- $\beta$ for $24 \mathrm{~h}$ was associated with thicker stress fibers and more prominent cortical actin bundles, together with more prominent Tpbg localization to the tips of both stress fibers and cortical actin filaments (fig. 7c). Quantitative analysis of the intensity of Tpbg staining (fig. 7d) and Factin staining (fig. 7e) demonstrated that both increased in the presence of TGF- $\beta$.

TGF- $\beta$ functions by binding to the heteromeric complex of serine/threonine kinase, type I receptor (ALK5) and the type II receptors. We confirmed the expression of ALK5 in podocytes (fig. 7f). SB431542, an ALK5 inhibitor, inhibited Tpbg staining intensity (fig. $7 \mathrm{~g}$ ) and the rearrangement of actin filaments (fig. $7 \mathrm{~h}$ ) induced by TGF- $\beta$. Furthermore, in the presence of SB431542 alone, the immunofluorescence intensity of Tpbg (fig. 7i) and F-actin (fig. 7j) decreased. Taken together, these results suggest that TGF- $\beta$ promotes Tpbg expression in differentiated podocytes, with localization to stress fibers and cortical actin.

\section{Tpbg Caused Actin Cytoskeletal Reorganization and Promoted Podocyte Migration}

We next carried out functional studies to directly assess the contribution of Tpbg to organization of the podocyte actin-based cytoskeleton and to podocyte migration. In differentiated podocytes transfected with control plasmid, stress fibers were maintained. In contrast, stress fiber formation was inhibited in podocytes transfected with full-length Tpbg (fig. 8a).

To confirm Tpbg knockdown, we demonstrated that podocytes transfected with si-Tpbg expressed less Tpbg mRNA compared to control podocytes transfected with si-scramble (fig. $8 b$ ). In the absence of TGF- $\beta$, no significant difference in wound closure rate at $24 \mathrm{~h}$ was observed between control and Tpbg knockdown podocytes. TGF- $\beta$ treatment promoted podocyte migration in control cells, but this effect was nearly abrogated in the presence of si-Tpbg (fig. 8c). We confirmed the result of knockdown experiments with wound closure assay by using three Tpbg siRNAs (si-Tpbg-1, -2 and -3; online suppl. fig. 2). We show the representative data in figure 8 . These results demonstrate that Tpbg modifies the phenotype of podocytes, including cell motility and actin cytoskeletal arrangement. 


\section{Discussion}

The present study had several principal findings. First, Tpbg expression was increased mainly in injured podocytes concurrently with mesangial cell proliferation in Thyl GN. The expression of podocyte proteins, which are required for maintaining the slit diaphragm structure, decreases in experimental podocyte injury models [18] and in human nephrotic syndrome [19]. Podocyte injury is a frequent finding in glomerulonephritis $[2,4,6,20$, 21]. Humoral [6] and/or mechanical $[5,22]$ factors derived from proliferating mesangial cells are thought to damage the podocytes. We focused on the effect of TGF$\beta$ as a possible mediator linking glomerular injury and Tpbg expression. As previously reported [23], we found an increase in the expression of TGF- $\beta$ mRNA concurrent with mesangial proliferation and increased Tpbg expression. In human carcinomas and embryonic stem cells, Tpbg overexpression is associated with reorganization of the actin cytoskeleton, decreased cell adhesion and increased cell motility [9-14, 24]. Taken together, these findings suggest that in the setting of glomerulonephritis, TGF- $\beta$ upregulates Tpbg expression in glomerular cells, particularly podocytes, which leads to alteration in the podocyte cytoskeleton, foot process architecture, adhesion to the glomerular basement and mobility. In vitro experiments using podocytes produced additional findings that lend support to this model.

Second, we found that Tpbg localization into focal adhesions was concurrent with the dynamic reorganization of stress fibers regulated by the RhoA signal $[25,26]$. Focal adhesions are specialized sites of cell attachment to the extracellular matrix [27], and are dynamic structures that assemble, disperse and recycle as cells migrate through cross-talk between Rho family GTPase, integrin and numerous adhesion proteins. The foot process architecture of podocytes critically depends on integrin-mediated cell-glomerular basement membrane interaction at focal adhesions. We considered that the tight regulation of focal adhesion assembly and disassembly is essential for the stability of podocyte architecture and that imbalance of focal adhesion proteins causes the impairment of contractile actin filaments. $\alpha_{3}$-integrin-deficient podocytes are unable to form mature foot processes, suggesting the importance of outside-in signaling [28]. Integrinlinked kinase, a focal adhesion kinase, plays a crucial role in podocyte adhesion, morphology and survival, and activation of integrin-linked kinase activation causes podocyte damage [29]. Cathepsin L, a lysosomal protease, is upregulated in a puromycin aminonucleoside-induced nephrosis model and modulates podocyte migration by interacting with $\alpha_{3}$-integrin [30]. Tpbg might modulate the regulation of focal adhesions when at low levels under normal conditions. In contrast, Tpbg overexpression and localization under pathological conditions might disassemble focal adhesions, which causes the motile phenotype of podocytes.

Third, we found that TGF- $\beta$ promoted Tpbg expression and localization at focal adhesions and that Tpbg modified actin organization and motility regulated by TGF- $\beta$. TGF- $\beta$ mediates podocyte injuries in many types of kidney disease [31-33]. Foot process effacement, which is observed in injured podocytes, requires a precise interplay of multiple cellular functions including structural alterations of the cytoskeleton, movement of foot processes over the basement membrane and reconstruction of the slit diaphragm [34]. There has been evidence that foot process effacement of podocytes is a migratory event. Lee et al. [35] reported that TGF- $\beta$ increases podocyte motility and albumin permeability through the MCP-1/ CCR2 pathway. Wei et al. [36] reported that urokinase receptor signaling is required to activate integrin in podocytes, promoting cell motility and activation of Cdc42 and Racl. The present data raise the possibility that, in response to increased TGF- $\beta$, Tpbg protein accumulates and localizes to focal adhesions, where it alters cytoskeleton function and enhances podocyte motility.

In conclusion, our study demonstrates that in response to TGF- $\beta$, Tpbg upregulation at focal adhesions enhances motility and might be involved in the cytoskeleton rearrangement in proliferative glomerulonephritis. Tpbg may be a novel therapeutic target to limit glomerular injury in proliferative glomerulonephritis.

\section{Acknowledgments}

We greatly appreciate the gift of conditionally immortalized murine podocytes from Dr. Peter Mundel (University of Miami). We would like to give our thanks to Naoto Kobayashi (Ehime University School of Medicine) and Akemi Shono (Kyoto University) for technical advice; Hideo Uchiyama (Taigenkai Hospital), Akiko Sakurai (Tokushima University Hospital), and Michael Hann (Florida International University) for technical assistance.

This study was supported by the Kidney Foundation, Japan (JKFB09-41), Mitsui Sumitomo Welfare Foundation and Grantin-Aid for Scientific Research (19590973) and the NIDDK Intramural Research Program. 


\section{References}

$\checkmark 1$ Kriz W, LeHir M: Pathways to nephron loss starting from glomerular diseases - insights from animal models. Kidney Int 2005;67: 404-419.

$\checkmark 2$ Hara M, Yanagihara T, Takada T, Itoh M, Matsuno M, Yamamoto T, Kihara I: Urinary excretion of podocytes reflects disease activity in children with glomerulonephritis. Am J Nephrol 1998;18:35-41.

3 Morioka Y, Koike H, Ikezumi Y, Ito Y, Oyanagi A, Gejyo F, Shimizu F, Kawachi $\mathrm{H}$ : Podocyte injuries exacerbate mesangial proliferative glomerulonephritis. Kidney Int 2001;60:2192-2204.

$\checkmark 4 \mathrm{Yu}$ D, Petermann A, Kunter U, Rong S, Shankland SJ, Floege J: Urinary podocyte loss is a more specific marker of ongoing glomerular damage than proteinuria. J Am Soc Nephrol 2005; 16:1733-1741.

$\checkmark 5$ Kriz W, Hackenthal E, Nobiling R, Sakai T, Elger M, Hahnel B: A role for podocytes to counteract capillary wall distension. Kidney Int 1994;45:369-376.

-6 Lai KN, Leung JC, Chan LY, Saleem MA, Mathieson PW, Tam KY, Xiao J, Lai FM, Tang SC: Podocyte injury induced by mesangialderived cytokines in IgA nephropathy. Nephrol Dial Transplant 2009;24:62-72.

$\checkmark 7$ Campean V, Karpe B, Haas C, Atalla A, Peters H, Rupprecht H, Liebner S, Acker T, Plate $\mathrm{K}$, Amann K: Angiopoietin 1 and 2 gene and protein expression is differentially regulated in acute anti-thyl.1 glomerulonephritis. Am J Physiol Renal Physiol 2008;294:F1174F1184.

$>8$ Hole N, Stern PL: A 72 kD trophoblast glycoprotein defined by a monoclonal antibody. Br J Cancer 1988;57:239-246.

>9 Southall PJ, Boxer GM, Bagshawe KD, Hole N, Bromley M, Stern PL: Immunohistological distribution of 5T4 antigen in normal and malignant tissues. Br J Cancer 1990;61:8995.

-10 Myers KA, Rahi-Saund V, Davison MD, Young JA, Cheater AJ, Stern PL: Isolation of a cDNA encoding 5T4 oncofetal trophoblast glycoprotein. An antigen associated with metastasis contains leucine-rich repeats. J Biol Chem 1994;269:9319-9324.

- 11 Carsberg CJ, Myers KA, Evans GS, Allen TD, Stern PL: Metastasis-associated 5T4 oncofoetal antigen is concentrated at microvillus projections of the plasma membrane. J Cell Sci 1995; 108:2905-2916.

>12 Carsberg CJ, Myers KA, Stern PL: Metastasis-associated 5T4 antigen disrupts cell-cell contacts and induces cellular motility in epithelial cells. Int J Cancer 1996;68:84-92.

13 Ward CM, Barrow K, Woods AM, Stern PL: The 5T4 oncofoetal antigen is an early differentiation marker of mouse ES cells and its absence is a useful means to assess pluripotency. J Cell Sci 2003;116:4533-4542.
14 Ward CM, Eastham AM, Stern PL: Cell surface 5T4 antigen is transiently upregulated during early human embryonic stem cell differentiation: effect of 5T4 phenotype on neural lineage formation. Exp Cell Res 2006;312: 1713-1726.

15 Eastham AM, Spencer H, Soncin F, Ritson S, Merry CL, Stern PL, Ward CM: Epithelialmesenchymal transition events during human embryonic stem cell differentiation. Cancer Res 2007;67:11254-11262.

16 Edlund S, Landstrom M, Heldin CH, Aspenstrom P: Transforming growth factor-betainduced mobilization of actin cytoskeleton requires signaling by small GTPases Cdc42 and RhoA. Mol Biol Cell 2002;13:902-914.

17 Bakin AV, Safina A, Rinehart C, Daroqui C, Darbary H, Helfman DM: A critical role of tropomyosins in TGF-beta regulation of the actin cytoskeleton and cell motility in epithelial cells. Mol Biol Cell 2004;15:46824694.

18 Luimula P, Ahola H, Wang SX, Solin ML, Aaltonen P, Tikkanen I, Kerjaschki D, Holthofer H: Nephrin in experimental glomerular disease. Kidney Int 2000;58:14611468.

19 Doublier S, Salvidio G, Lupia E, Ruotsalainen V, Verzola D, Deferrari G, Camussi G: Nephrin expression is reduced in human diabetic nephropathy: evidence for a distinct role for glycated albumin and angiotensin II Diabetes 2003;52:1023-1030.

20 Han GD, Koike H, Nakatsue T, Suzuki K, Yoneyama H, Narumi S, Kobayashi N, Mundel P, Shimizu F, Kawachi H: IFN-inducible protein-10 has a differential role in podocyte during Thy 1.1 glomerulonephritis. J Am Soc Nephrol 2003;14:3111-3126.

21 Sawai K, Mukoyama M, Mori K, Kasahara M, Koshikawa M, Yokoi H, Yoshioka T, Ogawa Y, Sugawara A, Nishiyama H, Yamada S, Kuwahara T, Saleem MA, Shiota K, Ogawa O, Miyazato M, Kangawa K, Nakao K: Expression of CCN1 (CYR61) in developing, normal, and diseased human kidney. Am Physiol Renal Physiol 2007;293:F1363F1372.

-22 Endlich N, Kress KR, Reiser J, Uttenweiler D, Kriz W, Mundel P, Endlich K: Podocytes respond to mechanical stress in vitro. J Am Soc Nephrol 2001;12:413-422.

23 Okuda S, Languino LR, Ruoslahti E, Border WA: Elevated expression of transforming growth factor-beta and proteoglycan production in experimental glomerulonephritis. Possible role in expansion of the mesangial extracellular matrix. J Clin Invest 1990; 86:453-462.

24 Starzynska T, Rahi V, Stern PL: The expression of $5 \mathrm{~T} 4$ antigen in colorectal and gastric carcinoma. Br J Cancer 1992;66:867-869.
25 Togawa A, Miyoshi J, Ishizaki H, Tanaka M, Takakura A, Nishioka H, Yoshida H, Doi T, Mizoguchi A, Matsuura N, Niho Y, Nishimune Y, Nishikawa S, Takai Y: Progressive impairment of kidneys and reproductive organs in mice lacking Rho GDIalpha. Oncogene 1999; 18:5373-5380.

$\checkmark 26$ Asanuma K, Yanagida-Asanuma E, Faul C, Tomino Y, Kim K, Mundel P: Synaptopodin orchestrates actin organization and cell motility via regulation of RhoA signalling. Nat Cell Biol 2006;8:485-491.

27 Sastry SK, Burridge K: Focal adhesions: a nexus for intracellular signaling and cytoskeletal dynamics. Exp Cell Res 2000;261: 25-36.

28 Kreidberg JA: Functions of alpha3beta1 integrin. Curr Opin Cell Biol 2000;12:548-553.

29 Teixeira Vde P, Blattner SM, Li M, Anders HJ, Cohen CD, Edenhofer I, Calvaresi N, Merkle M, Rastaldi MP, Kretzler M: Functional consequences of integrin-linked kinase activation in podocyte damage. Kidney Int 2005; 67:514-523.

30 Reiser J, Oh J, Shirato I, Asanuma K, Hug A, Mundel TM, Honey K, Ishidoh K, Kominami E, Kreidberg JA, Tomino Y, Mundel P: Podocyte migration during nephrotic syndrome requires a coordinated interplay between cathepsin 1 and alpha3 integrin. J Biol Chem 2004;279:34827-34832.

>31 Kopp JB, Factor VM, Mozes M, Nagy P, Sanderson N, Bottinger EP, Klotman PE, Thorgeirsson SS: Transgenic mice with increased plasma levels of TGF-beta 1 develop progressive renal disease. Lab Invest 1996;74: 991-1003

>32 Schiffer M, Bitzer M, Roberts IS, Kopp JB, ten Dijke P, Mundel P, Bottinger EP: Apoptosis in podocytes induced by TGF-beta and Smad7. J Clin Invest 2001;108:807-816.

-33 Li Y, Kang YS, Dai C, Kiss LP, Wen X, Liu Y: Epithelial-to-mesenchymal transition is a potential pathway leading to podocyte dysfunction and proteinuria. Am J Pathol 2008; 172:299-308.

>34 Somlo S, Mundel P: Getting a foothold in nephrotic syndrome. Nat Genet 2000;24:333335 .

35 Lee EY, Chung CH, Khoury CC, Yeo TK, Pyagay PE, Wang A, Chen S: The monocyte chemoattractant protein-1/CCR2 loop, inducible by TGF-beta, increases podocyte motility and albumin permeability. Am J Physiol Renal Physiol 2009;297:F85-F94.

>36 Wei C, Moller CC, Altintas MM, Li J, Schwarz K, Zacchigna S, Xie L, Henger A, Schmid H, Rastaldi MP, Cowan P, Kretzler M, Parrilla R, Bendayan M, Gupta V, Nikolic B, Kalluri R, Carmeliet P, Mundel P, Reiser J: Modification of kidney barrier function by the urokinase receptor. Nat Med 2008;14:55-63. 